\title{
The Impact of Preoperative Anaemia on Early Outcomes after Off-pump Coronary Artery Bypass Grafting
}

\author{
Sanjay Kumar Raha1 ${ }^{1}$ Smriti Kana Biswas ${ }^{2}$, Sorower Hossain ${ }^{3}$, Md Salahuddin Rahaman ${ }^{4}$, Khan Muhammad \\ Fahim Bin Enayet ${ }^{5}$,Md Kamrul Hasan ${ }^{6}$
}

\begin{abstract}
:
Introduction: In cardiac surgery, anaemia itself or combined with other risk factors has been found to be a major predictor for adverse outcome both preoperatively and postoperatively and even during extracorporeal circulation, but data about the specific tolerance of Coronary Artery Bypass Graft (CABG) patients for anaemia are conflicting and may in part be confounded by the effects of bypass surgery.

Objectives: This study was performed in the National Institute of Cardiovascular Diseases (NICVD) to observe whether the early outcomes of Off-Pump CABG (OPCAB)were affected by pre-operative haematocrit levels.

Methods: A total of 200 patients who underwent isolated OPCAB between January 2015 and December 2020 were retrospectively selected and purposively allocated into two groups: a) 100 patients having preoperative anaemia and b) $\mathbf{1 0 0}$ patients without preoperative anaemia. Preoperative, per-operative and early post-operative variables were recorded, compiled and compared.
\end{abstract}

Results: Preoperative characteristics were homogenously distributed between two groups other than haemoglobin level. Female patients had lower haemoglobin in each group. More patients of anaemic group required intraoperative and postoperative blood transfusion. The amount of blood loss and transfused blood products was also higher in anaemic patients. The ventilation time, length of ICU and post-operative hospital stay were significantly higher among anaemic patients. Among the post-operative complications, only the incidence of renal dysfunction was significantly higher among anaemic patients.

Conclusion:This study has showed that anaemic patients undergoing OPCAB had an increased risk of postoperative adverse events. Importantly, the extent of preexisting comorbidities substantially affected perioperative anaemia tolerance. Therefore, preoperative risk assessment, optimization and subsequent therapeutic strategies, such as blood transfusion, should take into account both the individual level of preoperative haemoglobin and the extent of concomitant risk factors.

Key Words: Anaemia, preoperative, OPCAB.

(Bangladesh Heart Journal 2021; 36(1): 47-54)

Introduction:

Coronary artery bypass surgery (CABG) is the most

disease. ${ }^{1}$ The success of surgery depends to some effective method for the treatment of ischemic heart extent on the elimination or improvement of risk factors

1. Associate Professor, Department of Cardiac Surgery, NICVD.

2. Medical Officer, 250 Bedded TB Hospital, Shyamoli.

3. Assistant Registrar, Department of Cardiac Surgery, NICVD.

4. Assistant Registrar, Department of Cardiac Surgery, NICVD.

5. Assistant Registrar, Department of Cardiac Surgery, NICVD.

6. Professor, Department of Cardiac Surgery, NICVD.

Address of Correspondence: Dr. Sanjay Kumar Raha, Department of Cardiac Surgery, National Institute of Cardiovascular Diseases NICVD, Dhaka-1207, Bangladesh. E-mail: drsanjayraha77yahoo.com, Contact: +8801720988629

DOI: https://doi.org/10.3329/bhj.v36i1.55517

Copyright $\odot 2017$ Bangladesh Cardiac Society. Published by Bangladesh Cardiac Society. This is an Open Access articles published under the Creative Commons Attribution-NonCommercial 4.0 International License (CC BY-NC). This license permits use, distribution and reproduction in any medium, provided the original work is properly cited and is not used for commercial purposes. 
or on taking measures against it. Some of these factors include advanced age, poor ventricular function, presence of diffuse coronary lesions, presence of poor respiratory or renal function, previous cardiac surgery, complicated surgery, and emergent surgical intervention. ${ }^{2}$

Low haemoglobin is progressively frequent sign in patients having coronary heart disease. ${ }^{3}$ About $51.5 \%$ patients with acute coronary syndrome in Indian population are anaemic. ${ }^{4}$ Similar prevalence of anaemia is expected in our population with ischemic heart disease. World Health Organization (WHO) defined anaemia as haemoglobin level $<13 \mathrm{gm} / \mathrm{dl}$ for adult male and $<12 \mathrm{gm} / \mathrm{dl}$ for adult female. ${ }^{5}$

Although anaemia is a potentially treatable condition prior to surgery, numerous studies have proved that low haemoglobin level is linked with higher rate of postoperative morbidity (specially renal and neurologic events) and mortality in patients suffering from congestive heart failure, ischemic heart disease particularly in acute coronary syndrome and in elderly population. 3,6,7 Diseased heart cannot raise cardiac output sufficiently; therefore, reduced level of haemoglobin, even when fully saturated, cannot supply enough oxygen throughout body. In theory, patients with coronary artery disease may tolerate anaemia well as long as the compensating mechanisms of the cardiovascular system are largely uncompromised and no extensive comorbidity exists. ${ }^{6}$ The impact of anaemia is confounded by blood transfusion during and after cardiac surgery. Blood transfusion increases postoperative morbidity and mortality. Anaemic patients need more blood transfusion. ${ }^{8}$ Several studies have found that, compared to non-anaemic patients, anaemic patients have significantly higher rate of operative mortality, prolonged ventilation, post-operative atrial fibrillation, post-operative renal dysfunction, stroke, deep sternal wound infection, perioperative blood transfusion, longer ICU stay and longer hospital stay after cardiac surgery. ${ }^{7,8,9,10,11}$

The commonly applied preoperative risk stratification models in cardiac surgery like EuroSCORE, STS, ACEF, do not realize preoperative low haemoglobin level as a possible predictor of unfavorable outcomes and two out of nineteen models of risk stratification admit anaemia as an unconventional predictor of mortality. ${ }^{3,12,13,14}$ Data from previous reports have disclosed preoperative anaemia to be self-sufficient predictor of unlucky outcomes in the form of morbidity and in-hospital death after coronary artery bypass graft (CABG) or valve surgery. $3,8,15$
Use of cardiopulmonary bypass during coronary artery bypass graft surgery has many adverse effects. Moreover, cardiopulmonary bypass increases the effect of anaemia and blood transfusion. ${ }^{16}$ Blood transfusion is usually required to increase haematocrit after weaning from cardiopulmonary bypass. Off-pump coronary artery bypass graft (OPCAB) surgery is a technique that avoids the adverse effects of cardiopulmonary bypass. There is evidence that OPCAB significantly reduces blood transfusion than on-pump coronary artery bypass grafting. ${ }^{17}$ So, in case of OPCAB surgery, impact of preoperative low haemoglobin level is minimally affected by blood transfusion and not affected by cardiopulmonary bypass.

The aim of this study was to investigate whether the routinely measured preoperative haematocrit level, affectsearly postoperative outcomes after OPCAB surgery.

\section{Materials and Methods:}

This retrospective observational study was performed in the department of Cardiac Surgery, National Institute of Cardiovascular Diseases (NICVD) from January 2015 and December 2020. 200 purposively selected patients who underwent elective off pump coronary artery bypass (OPCAB) graft surgery were allocated into two groups:

Group A: 100 patients having preoperative anaemia (haemoglobin level 9 to $13 \mathrm{gram} / \mathrm{dl}$ for male and 9 to 12 gram /dl for female).

Group B: 100 patients without preoperative anaemia (haemoglobin level 13 to $16 \mathrm{gram} / \mathrm{dl}$ for male and 12 to 15 gram /dl for female).

\section{Technique of OPCAB:}

Haemoglobin estimation was done within one week prior to surgery. All patients underwent standard off pump coronary artery bypass (OPCAB) graft surgery through median sternotomy. The internal thoracic artery, the radial artery, and the saphenous vein were harvested as appropriate with standard techniques. Then intravenous heparin $(100 \mathrm{IU} / \mathrm{kg}$ ) was given to maintain an ACT of 300 second before starting distal anastomoses. Regional myocardial immobilization was achieved with a suction stabilizer (Octopus) and apical suction device (Star Fish). Intracoronary shunts were used in most patients to maintain coronary flow, thereby reducing myocardial ischemia and at the sametime minimizing bleeding from the coronary arteriotomy. The left anterior descending (LAD) artery was revascularized first using left internal mammary/ thoracic artery (LIMA/ LITA). Proximal 
anastomoses were performed on the partially clamped ascending aortausing 6-0 continuous Prolene suture. Distal anastomoses were performed with continuous 70 or 8-0 polypropylene (Prolene) monofilament suture. After the procedure, heparin therapy was reversed with protamine sulfate in a 1:1 ratio. The leg, forearm, and chest wounds were closed and the patients were shifted to ICU. Total operation time and number of grafts were recorded.

\section{Postoperative Management:}

After surgery, all patients were transferred to the intensive care unit (ICU). Cardiac, respiratory, renal function and hourly blood loss were monitored meticulously. Extubation was done as early as possible while the patients fulfill the extubation criteria. Arterial blood gas, serum electrolytes and hematocrit estimation were done as per standard protocol. Haemodynamic and other parameters were managed according to the standard protocol. Blood transfusion trigger was haematocrit of $<26 \%$ or according to unit protocol. The amount of transfused blood or blood products were recorded. Serum creatinine was measured daily up to third postoperative day. Patients were on continuous ECG monitoring for 5 days and a check ECG was done prior to discharge to detect any arrhythmia. Neurological assessments were done on the first and second postoperative day.

\section{Data Collection:}

Data were collected using predesigned questionnaire and collection form. Data were analyzed and verified with statistical program for social sciences (SPSS) using student's t test, chi-square test, fisher's exact test where appropriate. The descriptive statistics used here were frequency, mean and standard deviation (SD) and compared using student's $t$ test. Categorical data were expressed as percentages and evaluated using chisquare or Fisher's exact probability test. The level of significance was 0.05 . Any $p$-value $<0.05$ was considered significant.

\section{Results:}

Patient characteristics are shown in the Table-1. The mean ages of the study sample were $60.3 \pm 7.86$ years for the group $A$ (with anaemia) and $58.9 \pm 6.79$ years for the group $B$ (without anaemia) showing no a significant difference $(p=0.2986 ;>0.05)$ in age distribution. In the study both sexes were homogenously distributed between the two groups but with clear male predominance ( $86 \%$ vs. $88 \%$; $p=0.766)$. The mean $\mathrm{BMI}$ were almost similar $\left(26.98 \pm 2.3 \mathrm{vs} .27 .2 \pm 2.14 \mathrm{~kg} / \mathrm{m}^{2}, p=\right.$
$0.5885 ;>0.05)$. The mean haemoglobin level among group A males was significantly lower than that among group B (11.7 \pm 1.18 vs. $13.6 \pm 0.55 ; p=<0.0001)$. The same was true for females $(10.2 \pm 0.58$ vs. $12.2 \pm 0.21$; $p=<0.0001)$. In our study, the commonest co-morbid factor was smoking in both groups $(48 \%$ vs. $51 \%$; $p=$ $0.6716)$. It was followed by hypertension ( $43 \%$ vs. $47 \%$; $p=0.5697)$, diabetes mellitus ( $38 \%$ vs. $41 \% ; p=0.6642)$ and dyslipidemia ( $34 \%$ vs. $35 \%$; $p=0.882$ ). Other comorbid factors were family history of CAD (10\% vs. $8 \%)$, past history of CVA ( $4 \%$ vs. $3 \%)$, COPD ( $11 \%$ vs. $9 \%)$, history of MI ( $45 \%$ vs. $47 \%)$, PVD ( $9 \%$ vs. $8 \%)$ and renal dysfunction ( $10 \%$ vs. $14 \%)$. All were almost identically distributed between the groups $(p>0.05)$. In the study the mean left ventricular ejection fraction (LVEF) was almost similar inboth groups $(46 \pm 5.7 \%$ vs. $47 \pm 7.2 ; p=$ 0.2775 ). Pre-operative angiographic study demonstrated that majority of the patients had triple vessel diseases (TVD) in each group ( $76 \%$ and $72 \%$ respectively). The rest had double vessel diseases (DVD) and left main diseases (LMD) which were similarly distributed among the two groups $(p>0.05)$. The distribution of NYHA and CCS angina classes between the two groups were also homogenous ( $p>0.05)$.

The mean operating times were similar in two groups $(268.5 \pm 33.5 v s$. $259.3 \pm 34.8 ; p=0.06)$. All the patients of both groups received left internal mammary artery (LIMA) and saphenous vein (SVG) as conduit. Radial artery was also used in some patients of both groups as second arterial conduit ( $24 \%$ vs. $28 \%$; $p=0.5190$ ). It is important to mention that all the left anterior descending arteries (LAD) of both groups were anastomosed to LIMA. Intraoperatively $74 \%$ anaemic and $56 \%$ non-anaemic patients required blood transfusion $(p=0.0076)$. Intra-operative hemoglobin level was significantly lower in group $A$ than group $B(9.1 \pm 1.1$ vs. $10.7 \pm 1.2 ; p=<0.000)$.

Post-operative transfusion of blood products was significantly higher among group A ( $56 \%$ vs. $28 \%$; $p=0.00006$ ). The mean amount of transfused blood products was also higher in group $\mathrm{A}(1085 \pm 45$ vs. $690 \pm$ $30 ; p=<0.0001)$. Although the 30 days mortality was similar among two groups, other post-operative variables like mechanical ventilation time, total bleeding, length of ICU stay, length of post-operative hospital stay were significantly higher among anaemic patients. Two patients of group $A$ and one of group $B$ required IABP post-operatively. Among the post-operative complications renal dysfunction was higher among anaemic patients ( $21 \%$ vs. $7 \% ; p=0.0043$ ). Other complications like low output syndrome (LOS) ( $6 \%$ vs. $2 \%$; $p=0.279$ ), reexploration for bleeding ( $3 \%$ vs. $1 \%$; $p=0.6212$ ), arrhythmia 
Table-I

Pre-Operative Characteristics of Patients with and without Anaemia

\begin{tabular}{|c|c|c|c|}
\hline Variables & Group $A(n=100)$ & Group B $(n=100)$ & $p$ Value \\
\hline Age, years $\#$ & $60.3 \pm 7.86^{*}$ & $58.9 \pm 6.79^{*}$ & $0.2986^{\text {ns }}$ \\
\hline Male, $\mathrm{n}(\%)^{¥}$ & $86(86)$ & $88(88)$ & $0.766^{\mathrm{ns}}$ \\
\hline $\mathrm{BMI}\left(\mathrm{kg} / \mathrm{m}^{2}\right)^{\#}$ & $26.98 \pm 2.3^{*}$ & $27.2 \pm 2.14^{*}$ & $0.5885^{\mathrm{ns}}$ \\
\hline \multicolumn{4}{|l|}{ Haemoglobin (gram/dl) } \\
\hline Male & $11.7 \pm 1.18^{*}$ & $13.6 \pm 0.55^{*}$ & $<0.0001^{s}$ \\
\hline Female & $10.2 \pm 0.58^{*}$ & $12.2 \pm 0.21^{*}$ & $<0.0001^{\mathrm{s}}$ \\
\hline Hypertension, n (\%) ${ }^{¥}$ & $43(43)$ & $47(47)$ & $0.5697^{\mathrm{ns}}$ \\
\hline Diabetes mellitus, $\mathrm{n}(\%)^{¥}$ & $38(38)$ & $41(41)$ & $0.6642^{\text {ns }}$ \\
\hline Smoking, $\mathrm{n}(\%)^{¥}$ & $48(48)$ & $51(51)$ & $0.6716^{\mathrm{ns}}$ \\
\hline Dyslipidemia, n (\%) $)^{¥}$ & $34(34)$ & $35(35)$ & $0.882^{\text {ns }}$ \\
\hline Family $\mathrm{H} / \mathrm{O}$ CAD, $\mathrm{n}(\%)^{¥}$ & $10(10)$ & $8(8)$ & $0.6212^{\text {ns }}$ \\
\hline Past H/O CVA, n (\%) & $4(4)$ & $3(3)$ & $1.000^{\text {ns }}$ \\
\hline COPD, n (\%) $)^{\#}$ & $11(11)$ & $9(9)$ & $0.6373^{\text {ns }}$ \\
\hline History of MI, n (\%) $)^{¥}$ & $45(45)$ & $47(47)$ & $0.7766^{\mathrm{ns}}$ \\
\hline PVD, $\mathrm{n}(\%)^{¥}$ & $9(9)$ & $8(8)$ & $0.7798^{\mathrm{ns}}$ \\
\hline Renal dysfunction, $\mathrm{n}(\%)^{¥}$ & $10(10.0)$ & $14(14)$ & $0.2841^{\mathrm{ns}}$ \\
\hline Arrhythmia, n (\%) $)^{¥}$ & $6(6)$ & $7(7)$ & $1.000^{\mathrm{ns}}$ \\
\hline LVEF (\%) & $46 \pm 5.7^{*}$ & $47 \pm 7.2^{*}$ & $0.2775^{\mathrm{ns}}$ \\
\hline NYHA class II or III, n (\%) & $16(16)$ & 14(14) & $0.6921^{\mathrm{ns}}$ \\
\hline CCS angina class III or IV, $\mathrm{n}(\%)^{¥}$ & $43(43)$ & $39(39)$ & $0.5652^{\text {ns }}$ \\
\hline LMD $¥$ & $10(10)$ & $12(12)$ & $0.6513^{\text {ns }}$ \\
\hline DVD $¥$ & $14(14)$ & $16(16)$ & $0.6921^{\mathrm{ns}}$ \\
\hline TVD $¥$ & $76(76)$ & $72(72)$ & $0.519^{n s}$ \\
\hline
\end{tabular}

* Data are presented as the mean \pm SD for continuous variable.

\# Student's t-Test, $¥$ Chi-square $\left(\chi^{2}\right)$ Test, I Fisher's Exact Test, $s=$ significant, $n s=$ Non-significant

CCS: Canadian Cardiovascular Society Angina Class; COPD: Chronic Obstructive Pulmonary Disease; CVA:Cerebrovascular Accident; DVD: Double Vessel Disease; LMD: Left Main Disease; MI: Myocardial Infarction; NYHA: New York Heart Association;PVD: Peripheral Vascular Disease; TVD: Triple Vessel Disease.

Table-II

Operative Data of Patients with and without Pre-operative Anaemia

\begin{tabular}{|c|c|c|c|}
\hline Variables & $\begin{array}{l}\text { Group A } \\
(n=100)\end{array}$ & $\begin{array}{c}\text { Group B } \\
(n=100)\end{array}$ & $p$ Value \\
\hline Total operating time, minutes ${ }^{\#}$ & $268.5 \pm 33.5^{*}$ & $259.3 \pm 34.8^{*}$ & $0.06^{\mathrm{ns}}$ \\
\hline \multicolumn{4}{|l|}{ Conduit used $¥$} \\
\hline LIMA, n (\%) & $100(100)$ & $100(100)$ & $1.000^{\text {ns }}$ \\
\hline Radial artery, n (\%) & $24(24)$ & $28(24)$ & $0.5190^{\mathrm{ns}}$ \\
\hline SVG, $n(\%)$ & $100(100)$ & $100(100)$ & $1.000 \mathrm{~ns}$ \\
\hline \multicolumn{4}{|l|}{ Graft distribution $¥$} \\
\hline LAD territory, $\mathrm{n}(\%)$ & $100(100)$ & $100(100)$ & $1.000 \mathrm{~ns}$ \\
\hline Circumflex territory, n (\%) & $93(93)$ & $97(97)$ & $0.1944 \mathrm{~ns}$ \\
\hline RCA territory, n (\%) & $85(85)$ & $83(83)$ & $0.6997^{\text {ns }}$ \\
\hline Intra-operative transfusion of blood products, $\mathrm{n}(\%)^{¥}$ & $74(74)$ & $56(56)$ & $0.0076^{s}$ \\
\hline Intra-operative lowest hemoglobin (gram/dl) \# & $9.1 \pm 1.1$ & $10.7 \pm 1.2$ & $<0.0001^{s}$ \\
\hline
\end{tabular}

* Data are presented as the mean \pm SD for continuous variable.

\# Student's t-Test, $¥$ Chi-square $\left(\chi^{2}\right)$ Test, $s=$ significant, $n s=$ Non-significant

LAD: Left Anterior Descending Artery; LIMA: Left Internal Mammary Artery; RCA: Right Coronary Artery; SVG: Saphenous Venous Graft. 
Table-III

Post-operative of Patients with and without Pre-operative Anaemia Data

\begin{tabular}{|c|c|c|c|}
\hline Variables & Group $A(n=100)$ & Group B $(n=100)$ & $p$ Value \\
\hline Post-operative transfusion of blood products & $56(56)$ & $28(28)$ & $0.00006^{s}$ \\
\hline Amount of blood products needed $(\mathrm{ml}) \#$ & $1085 \pm 45^{*}$ & $690 \pm 30^{*}$ & $<0.0001^{\mathrm{s}}$ \\
\hline $\begin{array}{l}30 \text { days mortality, } \mathrm{n}(\%)^{\pi} \\
0.6827^{\text {ns }}\end{array}$ & $4(4)$ & 2(2) & \\
\hline Ventilation time, hours $\#$ & $11.4 \pm 2.3^{*}$ & $8.6 \pm 3.5^{*}$ & $<0.0001^{\mathrm{s}}$ \\
\hline LOS or Prolonged inotropic support" & $6(6)$ & 2(2) & $0.279^{\text {ns }}$ \\
\hline $\begin{array}{l}\text { Postoperative IABPI } \\
1.000^{\text {ns }}\end{array}$ & $2(2)$ & 1(1) & \\
\hline Total bleeding (ml) \# & $602 \pm 85^{*}$ & $378 \pm 75^{*}$ & $<0.0001^{\mathrm{s}}$ \\
\hline Length of ICU stay (days) \# & $4.8 \pm 2.5$ & $3.4 \pm 2.2$ & $<0.0001^{\mathrm{s}}$ \\
\hline Length of post-operative hospital stay(days) \# & $10.3 \pm 1.3^{*}$ & $8.2 \pm 1.2^{*}$ & $<0.0001^{\mathrm{s}}$ \\
\hline Re-exploration for bleeding" & $3(3)$ & 1(1) & $0.6212^{\mathrm{ns}}$ \\
\hline Stroke & $3(3)$ & 1(1) & $0.6212^{\mathrm{ns}}$ \\
\hline Pulmonary complication $\llbracket$ & $4(4)$ & $2(2)$ & $0.6827^{\mathrm{ns}}$ \\
\hline Perioperative MIT & 2(2) & $2(2)$ & \\
\hline $1.000^{\mathrm{ns}}$ & & & \\
\hline Arrhythmia ${ }^{*}$ & $10(10)$ & $8(8)$ & $0.6212^{\mathrm{ns}}$ \\
\hline Surgical site infection $\pi$ & $6(6)$ & $4(4)$ & $0.7475^{\mathrm{ns}}$ \\
\hline Renal dysfunction $¥$ & $21(21)$ & $7(7)$ & $0.0043^{\mathrm{s}}$ \\
\hline
\end{tabular}

* Data are presented as the mean \pm SD for continuous variable.

\# Student's t-Test, $¥$ Chi-square $\left(\chi^{2}\right)$ Test, ๆ Fisher's Exact Test, $s=$ significant, $n s=$ Non-significant

IABP: Intra-aortic Balloon Pump; ICU: Intensive Care Unit; LOS: Low Output Syndrome; MI: Myocardial Infarction.

( $10 \%$ vs. $8 \% ; p=0.6212)$, peri-operative $\mathrm{Ml}(2 \%$ vs. 25 $p=1.000)$, stroke(3\% vs. $1 \% ; p=0.6212)$, pulmonary complications ( $4 \%$ vs. $2 \%$; $p=0.6827)$, surgical site infection ( $6 \%$ vs. $4 \% ; p=0.7475)$ were homogenously distributed among the two groups.

\section{Discussion:}

This study is unique in evaluating the effect of preoperative anaemia on post-operative outcomes in patients undergoing exclusively isolated off-pump CABG. Previous studies have reported several advantages of off-pump CABG compared with conventional CABG, including less intra-operative haemodilution. During CABG with cardiopulmonary bypass, the crystalloid priming solution and cardioplegia solution may decrease the haemoglobin concentration, increase haemodilutional anaemia, and result in worse postoperative outcomes. The associated morbidities may occur more frequently in patients with pre-operative anaemia. Moreover, although the pre-operative anaemia may allow for a period of adaptation, haemodilutional anaemia causes a sudden change in tissue oxygenation. All these factors render patients with anaemia at higher risk of adverse events when they undergo CABG with cardiopulmonary bypass. In patients undergoing offpump CABG, the effects of haemodilution with crystalloid priming solution and cardioplegic solution are eliminated.

According to the pre-operative characteristics the groups were comparable other than haemoglobin concentrations. Female patients had lower haemoglobin than male patients in each group. Kumar et al. ${ }^{2}$ and Karkouti et al. ${ }^{6}$ in their studies showed similar findings. However, Matsuda et al. ${ }^{19}$ in their retrospective observational study showed that patients with preoperative anaemia had more comorbidities than patients without pre-operative anaemia; patients with pre-operative anaemia were older and more likely to be female, and had a smaller body surface area, lower left ventricular ejection fraction, and greater renal dysfunction.

Although total operative time and graft distribution were similar among anaemic and non-anaemic groups, more patients of anaemic group required intra-operative blood transfusion ( $74 \%$ vs. $56 \%$ ) as their mean haemoglobin level was lower. The ventilation time, length of ICU and post-operative hospital stay were significantly higher 
among anaemic patients. Kumar et al. ${ }^{2}$ in their study found that post-operative drainage, the need for blood and blood product transfusion, and length of ICU and hospital stay were statistically higher in low-haematocrit group Matsuda et al. ${ }^{19}$ found that in the post-operative period more patients with pre-operative anaemia required blood transfusion and the amount of blood loss and blood products transfused were also higher in those patients. It has been suggested that peri-operative RBC transfusion negatively affects outcomes after cardiac surgery. More recently, Zhang and colleagues ${ }^{20}$ demonstrated that in patients who underwent CABG within 21 days of myocardial infarction, pre-operative anaemia was not associated with mortality or major adverse events; however, intraoperative RBC transfusion predicted major adverse events. In a study by Surgenor et al. ${ }^{21} 36 \%$ of patients received one to two units of packed cell transfusion; among them, $43 \%$ were transfused intraoperatively, and the rest were post-operatively transfused, and the mortality rate was found to be $16 \%$ higher in patients receiving transfusion compared to those who did not receive it.

Matsudaet al. ${ }^{19}$ showed that patients with intra-operative RBC transfusion had worse outcomes than patients without intra-operative RBC transfusion. The primary purpose of RBC transfusion is to increase oxygen delivery, which is determined by cardiac output and arterial oxygen saturation, the latter being dependent on the haemoglobin level. However, the Transfusion Requirements after Cardiac Surgerytrial conducted by Hajjar et al. ${ }^{22}$ compared a restrictive transfusion strategy $(<24 \%)$ with a liberal transfusion strategy $(<30 \%)$, reported no difference in the primary end point (combined outcome of 30-day mortality and severe morbidity) between the groups. The restrictive transfusion strategy did not result in reduced oxygen availability to cells, as demonstrated by similar lactate levels in both groups. The adverse effects of RBC transfusion include acute haemolytic and non-haemolytic reactions; transmission of viral and bacterial diseases, transfusion-related acute lung injury, transfusion-related acute kidney injury, transfusion-related circulatory overload and immunosuppression. The decision to transfuse should be individualized and based on a rational approach that accounts for physiologic variables in addition to the haemoglobin level.

Among the post-operative complications, we found the incidence of renal dysfunction was higher among anaemic group. Although the actual incidences of operative mortality, stroke, peri-operative Ml, arrhythmia, pulmonary complications and infective complications were higher among anaemic patients these were not statistically significant. A large study with greater sample size might show actual findings. Karkoutiet al. ${ }^{6}$ found that renal dysfunction was an important peri-operative pathophysiological factor for adverse outcome, being both a cause for and a result of pre-operative anaemia. It appears that the renal system especially with history of dysfunction was more sensitive than other organs to a temporary relative haemoglobin deficiency, thereby acting as a particularly sensitive and early indicator of pending ischemic injury to vital organs. Ranucci et al. ${ }^{8}$ found that patients with pre-operative haematocrit levels $<35 \%$ developed more than three-fold of major morbidities when compared to patients with pre-operative haematocrit levels of $>42 \%$ circulation Karkoutiet al. ${ }^{6}$ showed that pre-operative anaemia was associated with an increase of all post-operative adverse events, starting at haemoglobin level $<11 \mathrm{gm} / \mathrm{dl}$ in a dose-dependent fashion.Specially low pre-operative haemoglobin was an independent predictor for post-operative renal and central nervous system outcome, and the association with increased cardiac adverse events was caused by concomitant risk factors prevalent in anaemic patients. The extent of comorbidities substantially amplified the adverse effects of low pre-operative haemoglobin, which in turn was a significant marker for severe underlying diseases and comorbidities. Matsuda et al. ${ }^{19}$ in their study showed that patients with pre-operative anaemia had higher rates of low output syndrome, haemodialysis requirement, and the composite adverse outcome after isolated off-pump CABG than patients without preoperative anaemia. Patients with pre-operative anaemia also tended to have a higher rate of operative death, but this difference was not statistically significant. However, multivariate analyses showed that pre-operative anaemia was not an independent predictor of these post-operative outcomes. Rather old age was the only independent predictor of mortality; however, old age, high serum creatinine, prior myocardial infarction, and low ejection fraction were found to be significant independent predictors of mortality.

\section{Conclusion:}

The present study found that patients with preoperative anaemia had poorer outcomes after isolated off-pump CABG than patients without preoperative anaemia. Though the mortality among the anaemic patients was not significantly higher, a large scale study might have shown the exact scenario. The adverse outcomes might be related to associate comorbidities including age, smaller body surface area, lower glomerular filtration rate, congestive heart failure, non-elective surgery, and intraoperative blood transfusion. Cardiac surgery population is aging, and thus comorbid renal and central nervous system complications warrant increased 
attention because they play an important role in the decision to proceed with CABG. Awareness and optimization of risk factors prior to operation is essential for better outcomes and early recovery.

\section{Study Limitations:}

The present study has several limitations and those are as follows:

1. Sample size was small and patients were selected purposefully. There was possible selection bias in patients selected for off-pump CABG rather than CABG with cardiopulmonary bypass.

2. They were not randomly assigned to either group.

3. Intra-operative and post-operative blood transfusion was at the discretion of the surgeon or anaesthesiologist. So the relative importance of preoperative anaemia and intra-operative transfusion remained unclear.

4. The duration of follow up of this study was limited. Clinical outcomes were restricted to 30-days mortality. Patients were not followed up for medium and long-term results.

5. As a single institutional study the conclusions may not be applicable in general because of differences in practice patterns of other centres.

Other factors such as variations in surgical skill, patient difference in extent or distribution of coronary artery disease and echocardiography reports although unavoidable should also be considered.

\section{Recommendations:}

Despite the advances in cardiovascular medicine and cardiac surgical techniques, morbidity and mortality are still a problem after CABG. With increasing life expectancy, morbidity and mortality rates have also increased in elderly individuals. Due to this reason, preoperative assessment is extremely important to determine the risk factors. This study showed that anaemic patients undergoing OPCAB had an increased risk of postoperative adverse events. Importantly, the extent of pre-existing comorbidities substantially affected perioperative anaemia tolerance. By using risk stratification systems, operating surgeons, cardiac anaesthetists, ICU teams, patients' relatives, and the patients themselves become aware of what awaits them. We believe that preoperative haemoglobin level should be added to the risk scoring systems, which could be used to evaluate the patients' postoperative mortality risk and to predict the length of hospital stay and cost-efficacy.

\section{Acknowledgements:}

I owe my heartfelt gratitude and indebtedness to Professor Dr. Md. Kamrul Hasan, Professor, Department of Cardiac Surgery, NICVD for his active help, guidance and valuable suggestions.

\section{References:}

1. Pala AA, Taner T, Tatli AB, Ozsin KK, Yavuz S. The Effect of Pre-operative Hematocrit Level on Early Outcomes After Bypass Surgery. Cureus. 2020;12(4):e7811. DOI 10.7759/cureus. 7811.

2. Kumar S, Khurana NK, Awam I, Memon S, Memon MK, Sohail H, et al. The Effect of Preoperative Hematocrit Levels on Early Outcomes After Coronary Artery Bypass Graft. Cureus. 2021;13(1):e12733. DOI 10.7759/cureus.12733.

3. Egypt original :Sallam A, Hassan E. Effect of preoperative hemoglobin level on early outcomes in patients undergoing urgent coronary artery bypass grafting. Journal of the Egyptian Society of CardioThoracic Surgery. 2018;26:252-256.

4. Bhavanadhar P, Srinivasan VR, Oruganti SS, Adiraju KP. A prospective study on prevalence and causes of anaemia in patients with acute coronary syndrome. Journal of Clinical and Diagnostic Research. 2016;10(7):01-05.

5. BenoistDB, McLean E, Egli I, Cogswell M, editors. Worldwide prevalence of anaemia 1993-2005: WHO global database on anemia, vol. 4. Swizerland: WHO; 2008[Haemoglobin thresholds used to define anaemia].

6. Karkouti K, Wijeysundera DN, Beattie S. Risk Associated With Preoperative Anemia in Cardiac Surgery: A Multicenter Cohort Study. Circulation. 2008; $117: 478-484$

7. Williams ML, He X, Rakin JS, Slaughter MS, Gammie JS. (2013). Pre-Operative Hematocrit is a Powerful Predictor of Adverse Outcomes in Coronary Artery Bypass Graft Surgery: A Report from the Society of Thoracic Surgeons Adult Cardiac Surgery Database. The Annals of Thoracic Surgery.2013;96:1628-1634.

8. Ranucci M,Dedda DU, Castelvecchio S, Menicanti L, Frigiola A, Pelissero G. Surgical and Clinical Outcome Research Group (SCORE): Impact of Preoperative Anemia on Outcomes in Adult Cardiac Surgery: A Propensity-matched Analysis. Ann Thorac Surg. 2012;94:1134-42.

9. Bell ML, Grunwald GK, Baltz JH, McDonald GO, Bell $\mathrm{MR}$, Grover FI, et al. Does pre-operative hemoglobin independently predict short-term outcomes after coronary artery bypass graft surgery? Ann of Thorac Surg. 2008;86:1415-1423.

10. Dai L, Mick SL, McCrae KR, Houghtaling PL, Sabik $\mathrm{JF}$, Blackstone $\mathrm{EH}$, et al. Pre-operative Anemia in Cardiac Operation: Does Hemoglobin Tell the Whole Story? Ann of Thorac Surg. 2018;105: 100-107. 
11. Miceli A, Romeo F,Glauber M, Siena PMD, Caputo $M$, Angelini. Preoperative Anemia Increases Mortality and Postoperative Morbidity After Cardiac Surgery. Journal of Cardiaothoracic Surgery. 2014;9: 137-143.

12. Magovern JA, Sakert T, Magovern GJ, Benckart DH, Burkholder JA, LieblerGA, et al. A Model that Predicts Morbidity and Mortality after Coronary Artery Graft Surgery. J Am CollCardiol. 1996;28:1147-53.

13. Nilsson JU, Ohlsson, M, Thulin L, Hogland P, Nashef SA, Brant J. Comparison Of 19 Preoperative Risk Stratification Models in Open Heart Surgery. Eur Heart J. 2006;27:867-74.

14. Higgins TL, Estafanous FG, Loop FD, Beck GJ, Blum JM, Paranandi L. Stratification of Morbidity and Mortality Outcome by Preoperative Risk Factors In Coronary Artery Bypass Patients. A Clinical Severity Score. JAMA. 1992;267:2344-2348.

15. Zindrou D, Taylor KM, Bagger JP. Preoperative Haemoglobin Concentration and Mortality Rate after CABG. Lancet 2002;359:1747-1748.

16. Egypt 8 Bojar R., In: Bojar R, editor. Manual of Perioperative Care in Adult Cardiac Surgery. 5th ed. West Sussex:Wiley-Blackwell;2011.p.243-244.
17. Bojar R., In: Bojar R, editor. Manual of Peri-operative Care in Adult Cardiac Surgery. 5th ed. West Sussex:Wiley-Blackwell;2011.p.243-244.

18. Lamy A, Devereaux PJ, Prabhakaran D, Taggart DP, Hu S, Paolasso E. Off-Pump or On-Pump Coronary Artery Bypass Grafting at 30 Days. The New England Journal of Medicine. 2012;366:1489-1497.

19. Matsuda S, Fukui T, Shimizu J, Takao A, Takanashi $\mathrm{S}$, Tomoike H. Associations Between Preoperative Anemia and Outcomes After Off-Pump Coronary Artery Bypass Grafting. Ann Thorac Surg. 2013;95:854-61.

20. Zhang L, Hiebert B, Zarychanski R, Arora RC. Preoperative Anaemia Does Not Increase the Risks of Early Surgical Revascularization After Myocardial Infarction. Ann Thorac Surg. 2013;95:542-7.

21. Surgenor SD, Kramer RS, Olmstead EM. The Association of Perioperative Red Cell Transfusions and Decreased Long-term Survival after Cardiac Surgery. AnesthAnalg. 2009;108:1741-1746.

22. Hajjar LA, Vincent JL, Galas Fr. Transfusion requirements after cardiac surgery: the TRACS randomized control trial, JAMA. 2010;304:1559-67. 